\title{
STABILITY AND SEMIPOSITIVITY OF REAL MATRICES1
}

\author{
BY ABRAHAM BERMAN AND ROBERT C. WARD
}

Communicated by Alston S. Householder, September 16, 1976

The purpose of this note is to describe the interrelations of various degrees of stability and semipositivity for real square matrices.

A matrix $A$ is

(a) stable if there exists a positive definite matrix $X$ such that

$$
A X+X A^{T}
$$

is positive definite [4], [5];

(b) diagonally stable if $X$ in (1) may be taken to be diagonal [1]; and

(c) semipositive if there exists a positive vector $x$ such that $A x$ is positive [6]. We denote by $L, A$, and $S$ the classes of stable, diagonally stable, and semipositive matrices, respectively.

With each of these classes, we associate two superclasses denoted by $W A$, $W L, W S$ and $V W A, V W L, V W S$. A matrix $A \in W L(V W L)$ if there exists a positive definite (nonzero positive semidefinite) matrix $X$ such that (1) is positive semidefinite. The other four superclasses are defined similarly.

Let $I$ denote any of these nine classes. With each $I$ we associate subclasses using the following notation:

$A \in I S$ if every principal submatrix of $A$ is in $I$;

$A \in D I(I D)$ if $D A(A D)$ is in $I$ for every positive diagonal matrix $D$. ISD and DIS are defined similarly.

We also consider the classes $P, P_{0}$, and $P_{0}^{+}$defined as follows:

$A \in P$ if all its principal minors are positive;

$A \in \mathrm{P}_{0}$ if all its principal minors are nonnegative; and

$A \in \mathrm{P}_{0}^{+}$if $A \in \mathrm{P}_{0}$ and has at least one positive principal minor of each order [2], [3].

A study of the above classes indicates that there exist 24 distinct ones. The inclusion relations between them are described by the following directed graph having classes as vertices in which there is a sequence of directed edges from vertex $X$ to vertex $Y$ if and only if $X$ is contained in $Y$.

AMS (MOS) subject classifications (1970). Primary 15A48; Secondary 15A15, 15A1 8, 65F15, $93 \mathrm{D} 05$.

1 Research sponsored by the Union Carbide Corporation, Nuclear Division, Computer Sciences Division, Oak Ridge, Tennessee, under contract with the U.S. Energy Research and Development Administration. 


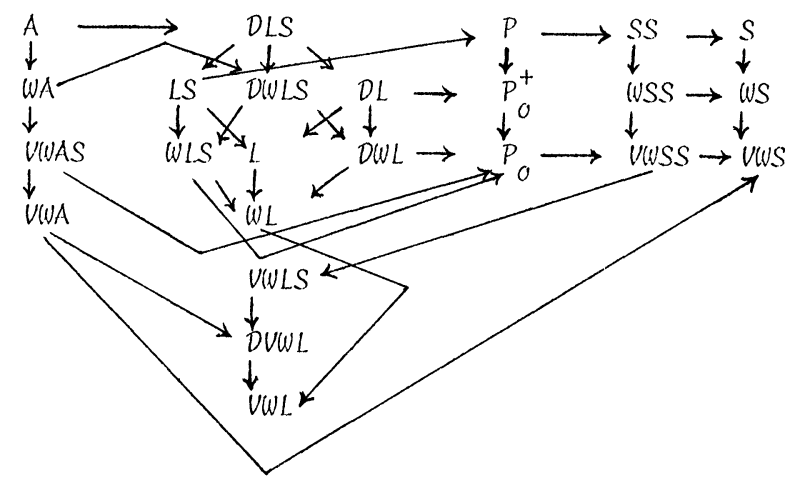

\section{REFERENCES}

1. G. P. Barker, A. Berman and R. J. Plemmons (1976), Positive diagonal solutions to the Lyapunov equations, Math. Res. Center Report \#76-087, Univ. of Wisconsin, Madison.

2. M. Fiedler and V. Pták (1962), On matrices with non-positive off-diagonal elements and positive principal minors, Czechoslovak Math. J. 12 (87), pp. 382-400. MR 26 \#134.

3. C. R. Johnson (1974), Second, third, and fourth order D-stability, J. Res. Nat. Bur. Standards Sect. B 78 pp. 11-13. MR 49 \#5042.

4. A. M. Lyapunov (1892), Problème général de la stabilité du mouvement, Comm. Math. Soc. Kharkow; English transl., Ann. of Math. Studies, no. 17, Princeton Univ. Press, Princeton, N.J.; Oxford Univ. Press, London, 1947. MR 9, 34.

5. O. Taussky, A remark on a theorem of Lyapunov, J. Math. Anal. Appl. 2 (1961), 105-107. MR 23 \#A1649.

6. J. Vandergraft (1972), Applications of partial orderings to the study of positive definiteness, monotonicity and convergence, SIAM J. Numer. Anal. 9, pp. 97-104. MR 46 \#9074.

UNION CARBIDE CORPORATION, NUCLEAR DIVISION, MATHEMATICS AND STATISTICS RESEARCH DEPARTMENT, COMPUTER SCIENCES DIVISION, P.O. BOX Y, BLDG. 9704-1, OAK RIDGE, TENNESSEE 37830 (Current address of R. C. Ward)

Current address (Abraham Berman): Department of Mathematics, Technion Israel Institute of Technology, Haifa, Israel 